\title{
Electron Testbeam Results for the ATLAS Liquid Argon Forward Calorimeter Prototype
}

\author{
M.I. Ferguson ${ }^{\text {a }}$, L. Harlow ${ }^{\text {b }}$, P. Loch* M. Qic, J. Rutherfoord, A. Savine, \\ L. Shaver, M. Shupe, J. Steinberg, C. Zeitnitz ${ }^{\mathrm{d}}$ \\ University of Arizona, Tucson, Arizona 85721, USA \\ and \\ D. Rahm \\ Brookhaven National Laboratory, Upton, New York 11973, USA \\ a now at University of California, Los Angeles, USA \\ ${ }^{\mathrm{b}}$ now at Synergy, Tucson, Arizona, USA \\ c on leave from Nanjing University, Nanjing, People's Republic of China \\ $\mathrm{d}$ now at University of Mainz, Mainz, Germany \\ ${ }^{*}$ corresponding author (e-mail: Peter.Loch@cern.ch)
}

March 5, 1997

\begin{abstract}
A novel liquid argon calorimeter concept with thin cylindrical shell gaps, meeting the required physics performance for the forward region $(|\eta|>3)$ in a high energy, high luminosity hadron collider experiment such as ATLAS, is described. A prototype for the electromagnetic section was built and tested in two electron testbeam runs at the Brookhaven AGS and at the CERN North Area in 1993, covering a total energy range from 2 to $200 \mathrm{GeV}$. Dependencies of the signal on the impact angle and varying inactive material in front of the calorimeter have been studied. Important performance requirements such as signal linearity better than $2 \%$, a sufficient energy resolution with a constant term of about $4 \%$ and a very good space resolution of about $0.6 \mathrm{~mm}$ have been demonstrated.
\end{abstract}

publ. in Nucl. Inst. and Meth. A 383 (1996) 399-408 


\section{Introduction}

Some of the most important physics to be reconstructed at future high energy, high luminosity hadron collider experiments such as ATLAS [1] at the LHC requires calorimetric coverage at least to pseudo-rapidities $|\eta| \sim 5$. This is certainly true not only for missing transverse energy measurements in events such as Higgs decays $H \rightarrow Z Z \rightarrow l \bar{l} \nu \bar{\nu}$ and SUSY, but also for jet tagging at high $|\eta|$ in electroweak channels such as $W W$ scattering [2]. The premier performance requirement for any calorimeter in the forward direction is therefore good transverse energy resolution with a significant contribution coming from the angular measurement for $|\eta| \gtrsim 4$.

Furthermore the forward region in a hadron collider detector is where particle densities and energies from the underlying minimum bias events are highest. At a luminosity of $10^{34} \mathrm{~cm}^{-2} \mathrm{~s}^{-1}$ for example one expects about $7 \mathrm{TeV}$ of energy deposited in a forward calorimeter $3<|\eta|<5$ in each bunch crossing every $25 \mathrm{~ns}$, at LHC design beam energies. Forward calorimeters will therefore be exposed to high radiation levels from electromagnetic and hadronic showers. These high particle energies and fluxes demand radiation hard materials and fast response to minimize pile-up.

Liquid argon (LAr) calorimetry is particularly attractive in such an environment: LAr and absorber metals are radiation hard, leading to long term stable operation. Fast charge collection to minimize pile-up and, even more, the low mobility of the ionized argon require small gaps of active medium on the order of only a few $100 \mu \mathrm{m}$, to avoid a luminosity dependent calibration due to positive charge build-up [3] and to suppress additional signal fluctuations originating from the same effect. A simple, practical solution to obtain precision narrow gaps in a sampling calorimeter is a tube/rod geometry, where the active gaps are tubular in shape (cylindrical shells). This geometry not only allows simple fabrication techniques, but also meets all physics requirements for the LHC.

A prototype of an electromagnetic forward calorimeter (FCal) with such an electrode geometry, using brass as absorber material, was designed and built at the University of Arizona in 1993. It was extensively studied in electron testbeams, first at Brookhaven National Laboratory (BNL) in the energy range from 2 to $8 \mathrm{GeV}$ in summer 1993 and later (October 1993) at $\mathrm{CERN}$ in the higher energy domain $(20$ to $200 \mathrm{GeV})$. The major goals of these measurements were the basic proof of principle and the determination of the performance parameters such as absolute charge collected, linearity of response, energy resolution, and spatial resolution. Here we report first experimental results along with comparisons to simulations.

In the first section of this note the mechanical and electrical design of the FCal prototype are described. The setup at the testbeam and simulation parameters are explained in the second section. Electronic calibration and data analysis strategies are discussed in the third section. Experimental results for signal linearity, spatial and energy resolution are finally given in section 5, together with detailed simulation results. This section is followed by conclusions and a brief outlook for further testbeam studies and expected applications in the ATLAS experiment. 


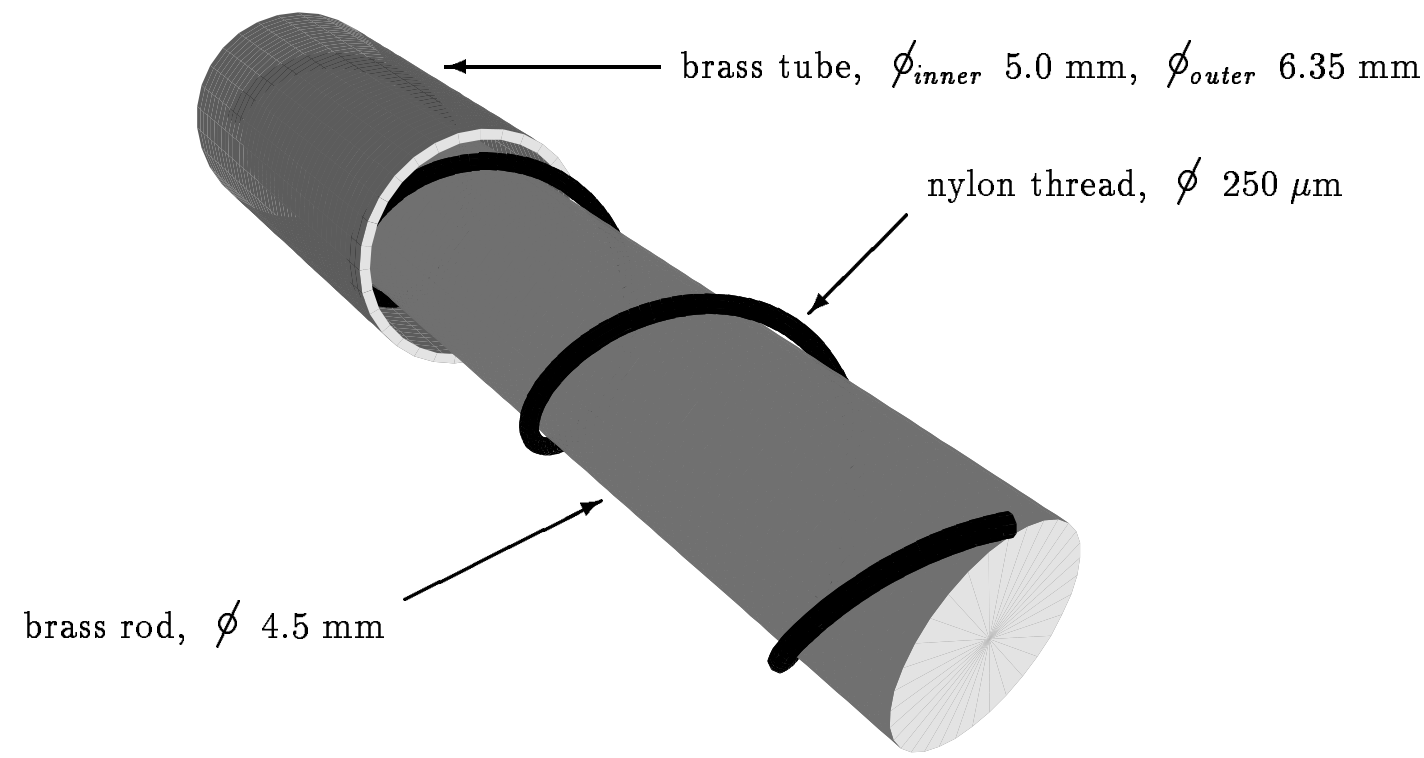

Figure 1: Rod and tube electrode assembly with the rod pulled out of the tube to show the parts. The LAr gap between tube and rod is maintained by the nylon thread which also electrically isolates the rod.

\section{Prototype Design and Electronics}

The thin gap tubular electrode structure is a departure from previous liquid argon calorimeter structures. Not only does it provide an economical solution to the radiation problem but it also allows the signal to be cleanly transferred from the electrode to the electronics.

\subsection{Prototype Design}

The design of the liquid argon/brass FCal prototype is intended to achieve optimal performance with respect to the projected physics, while at the same time emphasizing simple and mechanically robust fabrication techniques. The unit cell consists of a solid brass rod of 4.5 $\mathrm{mm}$ in diameter, inserted in a brass tube of inner diameter $5.0 \mathrm{~mm}$, resulting in a cylindrical gap of $250 \mu \mathrm{m}$ around the rod. The rod is fixed at both ends of the tube by small plastic caps, giving a sensitive depth of $25.4 \mathrm{~cm}$, out of $26.3 \mathrm{~cm}$ total. The caps are flat on the sides, so that the LAr can flow into the gap. The gap spacing is maintained by an insulating nylon thread wound around the rod, having the same diameter as the desired gap width, as seen in Fig. 1. For radiation hardness, the nylon threads will be replaced by quartz fibers in future prototypes and in the ATLAS FCal itself.

An electric field of about $1.2 \mathrm{kV} / \mathrm{mm}$ is applied across each gap to collect the ionization electrons in the argon. The signal is then fed out at the rear end of the rod and tube assembly. 


\section{FCal prototype specifications:}

Absorber material $\ldots \ldots \ldots \ldots \ldots \ldots \ldots \ldots \ldots \ldots \ldots$ yellow brass Outer diameter .............................. $180.0 \mathrm{~mm}$ Overall depth ............................... $276.9 \mathrm{~mm}$ Sensitive depth $\ldots \ldots \ldots \ldots \ldots \ldots \ldots \ldots .254 .0 \mathrm{~mm} \approx 14.4 X_{0} \approx 1.4 \lambda$ $d E / d x($ mip $)$ weighted sampling fraction $\ldots \ldots \ldots \ldots \ldots \ldots \ldots 1.5 \%$

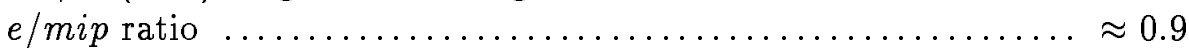

\section{Rod/tube electrode assembly specifications:}

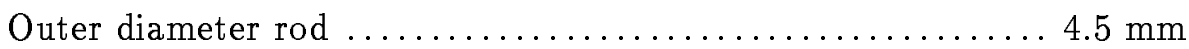

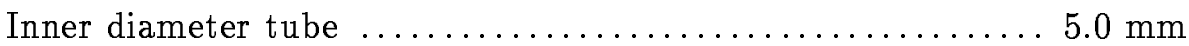

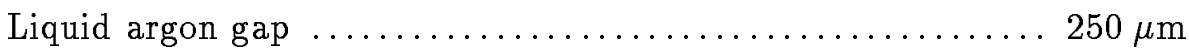

Capacitance ................................. $200 \mathrm{pF}$

Readout electrons per $\mathrm{GeV} \ldots \ldots \ldots \ldots \ldots \ldots \ldots \ldots \ldots \ldots 0.26 \cdot 10^{6}$

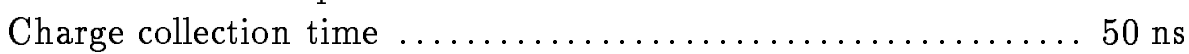

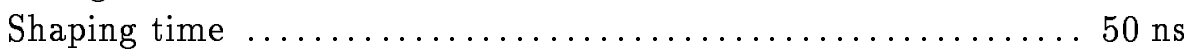

Effective gain at BNL $\ldots \ldots \ldots \ldots \ldots \ldots \ldots \ldots \ldots \ldots \approx 960 \mathrm{mV} / \mathrm{pC}$

Effective gain at CERN $\ldots \ldots \ldots \ldots \ldots \ldots \ldots \ldots \ldots \ldots \approx 48 \mathrm{mV} / \mathrm{pC}$

Table 1: Mechanical and electrical parameters of the FCal prototype. The e/mip ratio is calculated from electron shower simulations, as described in section 3.3, and is therefore dependent on the applied cuts and the quality of the geometry description.

The capacitance of each electrode is about $200 \mathrm{pF}$ when filled with LAr.

The complete calorimeter prototype is a cylinder $18 \mathrm{~cm}$ in diameter and $27.7 \mathrm{~cm}$ in overall depth. It consists of a stack of 9 round brass plates bolted together with two endplates and placed inside an outer sleeve which locates the endplates with respect to each other. A hexagonal array of holes is drilled in each plate. Those in the endplates are reamed to high precision to accurately locate the 374 rod and tube electrodes transversally and to establish the ground connection between them. The center-to-center distance between two adjacent electrodes is $7.5 \mathrm{~mm}$ (Fig. 2(a)). The longitudinal position of the tube/rod electrode inside the absorber is fixed by small springs on the front end of each unit, as shown in Fig. 2(b).

Of the 374 holes in the absorber matrix, only 192 were filled with electrodes. Pairs of electrodes were ganged for readout as shown in Fig. 2(a) yielding 96 electronic readout channels.

The overall depth of the prototype is about 14.4 radiation lengths $\left(X_{0}\right)$ and about $1.4 \mathrm{ab}$ sorption lengths $(\lambda)$. Electromagnetic showers are well contained transversely. The sensitive width is about 7.5 Molière radii $R_{M}$, where $1 R_{M} \approx 13.9 \mathrm{~mm}$. The sampling fraction for a minimum ionizing particle is about $1.5 \%$, while about $7.7 \%$ of the total volume is filled with LAr. Important physical parameters are listed in Table 1. 


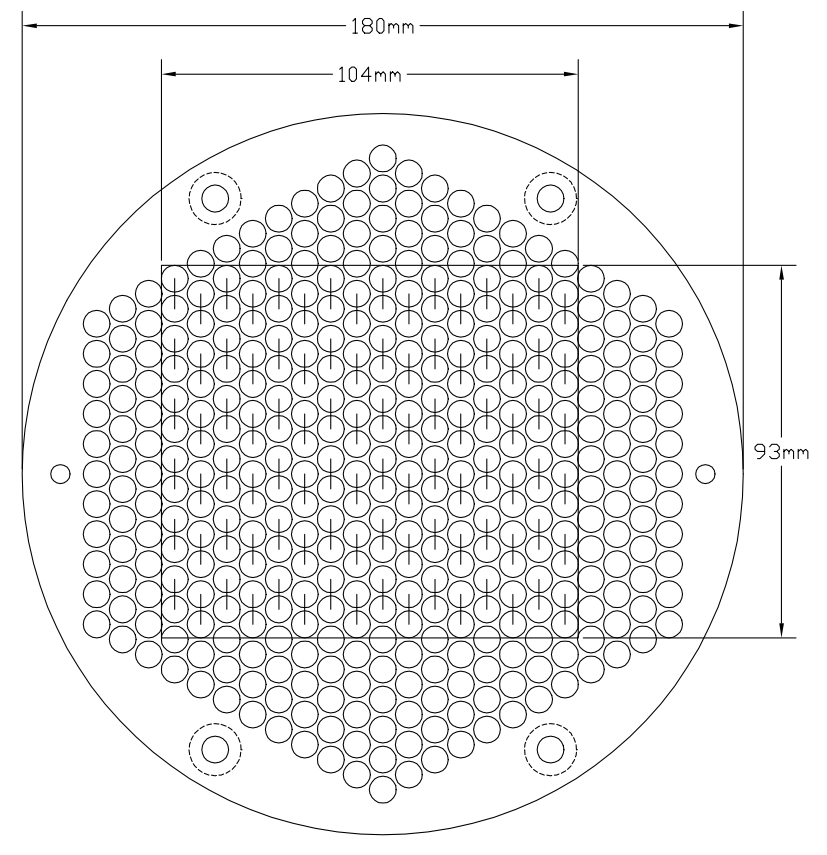

(a)

$276.9 \mathrm{~mm}$

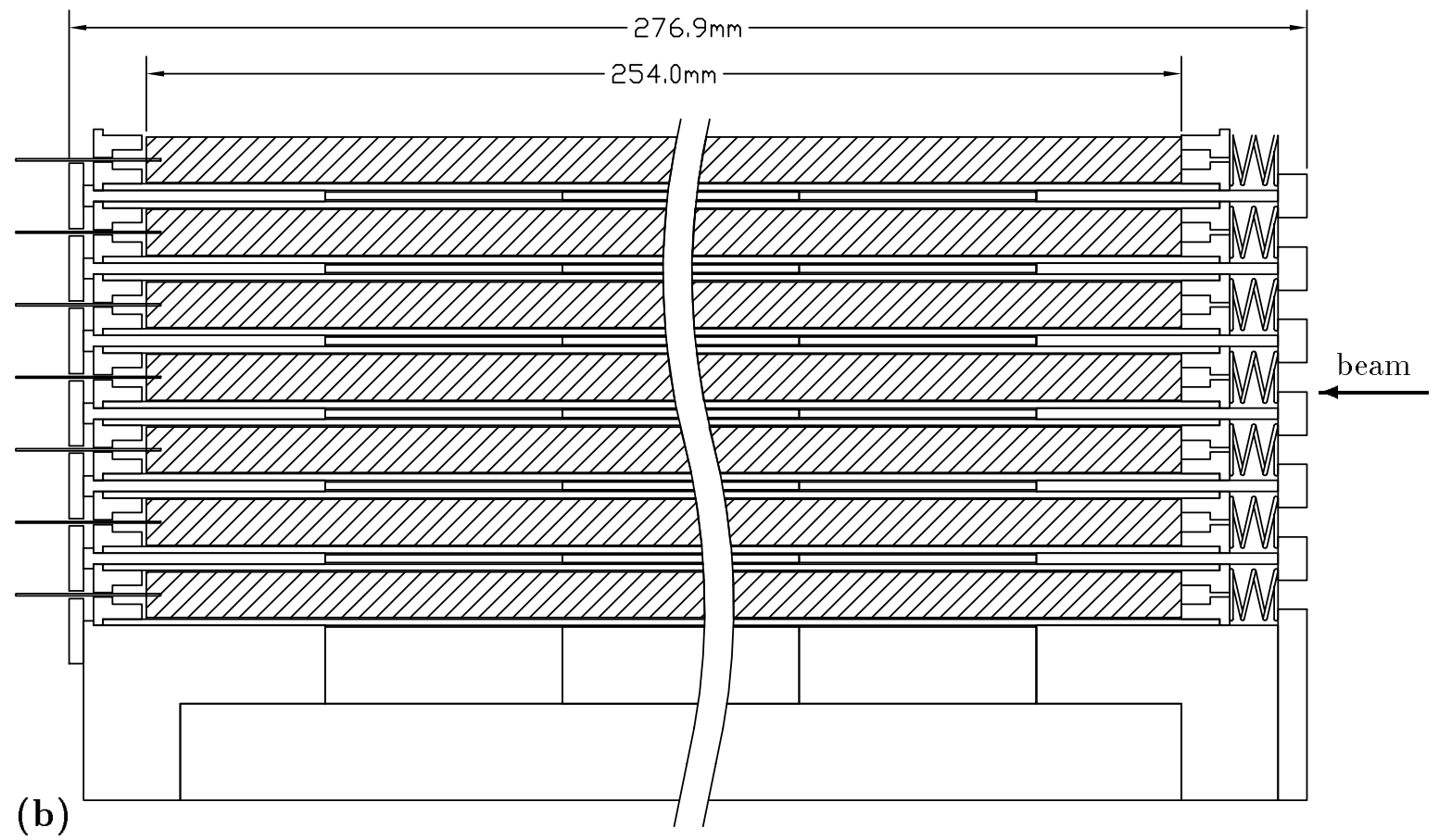

Figure 2: Transverse (a) and longitudinal (b) cross sections of the FCal prototype. The rectangular area in (a) shows the 192 tubes which are ganged together by pairs to form 96 electronic channels. 
The whole calorimeter is mounted on a support structure suspended below the top plate of a small cryostat, as shown in Fig. 3. A LAr purity monitor using a $\beta$ source (as described in [4]) is mounted on the same support. The monitor has an extra feedthrough in the top plate.

\subsection{Electronic Readout Chain}

The signals collected at the downstream end of the FCal prototype are fed out on 96 individual $50 \Omega$ coax cables which exit the cryostat via a common feedthrough in the top plate. A copper box is mounted on top of the feedthrough, holding a crate with six preamp motherboards and a preamp control board for electronic calibration purposes. Each motherboard has a charge pulse source and 16 individual preamp boards on it, holding the preamp itself and a shaper. The preamp design scheme follows the " $\emptyset \mathrm{T}$ " approach [5]. As the charge collection time is rather short (about $50 \mathrm{~ns}$ ), a shaping time of about $50 \mathrm{~ns}$ was chosen to avoid significant signal losses due to ballistic deficit effects. The effective gain of the preamps was adjusted to the electron energy ranges at BNL and CERN, respectively (see Table 1). The electronic noise of the chain is conservatively estimated at about 14000 electrons per channel rms. High voltage providing the electric field in the gap is applied via the signal lines (typically $300 \mathrm{~V}$ ), while the absorber matrix is grounded.

For each channel the amplified and shaped signal is sent to a track \& hold unit, converting the signal to a voltage level corresponding to the amplitude at the gate which is timed to the peak. This voltage is digitized by a LeCroy 4300B 11-bit FERA analog-to-digital converter (ADC), with a fixed gate of about $100 \mathrm{~ns}$. The granularity of the digitization was about 1250 electrons/ADC count at BNL, and about 25000 electrons/ADC count at CERN, corresponding to a primary energy granularity of about 5 and $100 \mathrm{MeV} / \mathrm{ADC}$ count, respectively. This obviously makes the determination of the electronic noise of the preamp and shaper units in the CERN setup problematic as the noise in this case is on average less than one ADC count. The observed pedestal fluctuations (typically $4-5$ counts) which were larger than the predicted electronics noise were due to second stage noise in the track $\&$ hold units.

\subsection{Data Acquisition and Monitoring}

The data acquisition system is based on a personal computer with an Intel 486 CPU, reading the digitized calorimeter signals and other event related data (counter signals, trigger words, scalers) via CAMAC. Particle and other events triggered during a beam spill are stored in the PC memory and dumped to a hard-disk after each spill. This system works reliably up to a peak event rate of more than $750 \mathrm{~Hz}$, with typical average rates of $200-400 \mathrm{~Hz}$, depending on the beam energies (quoted numbers observed at CERN). The total event size is 12716 -bit data words.

Additional scalers and monitor data such as cryostat temperature and pressure are read at out-of-spill times and stored synchronously with the event data. 


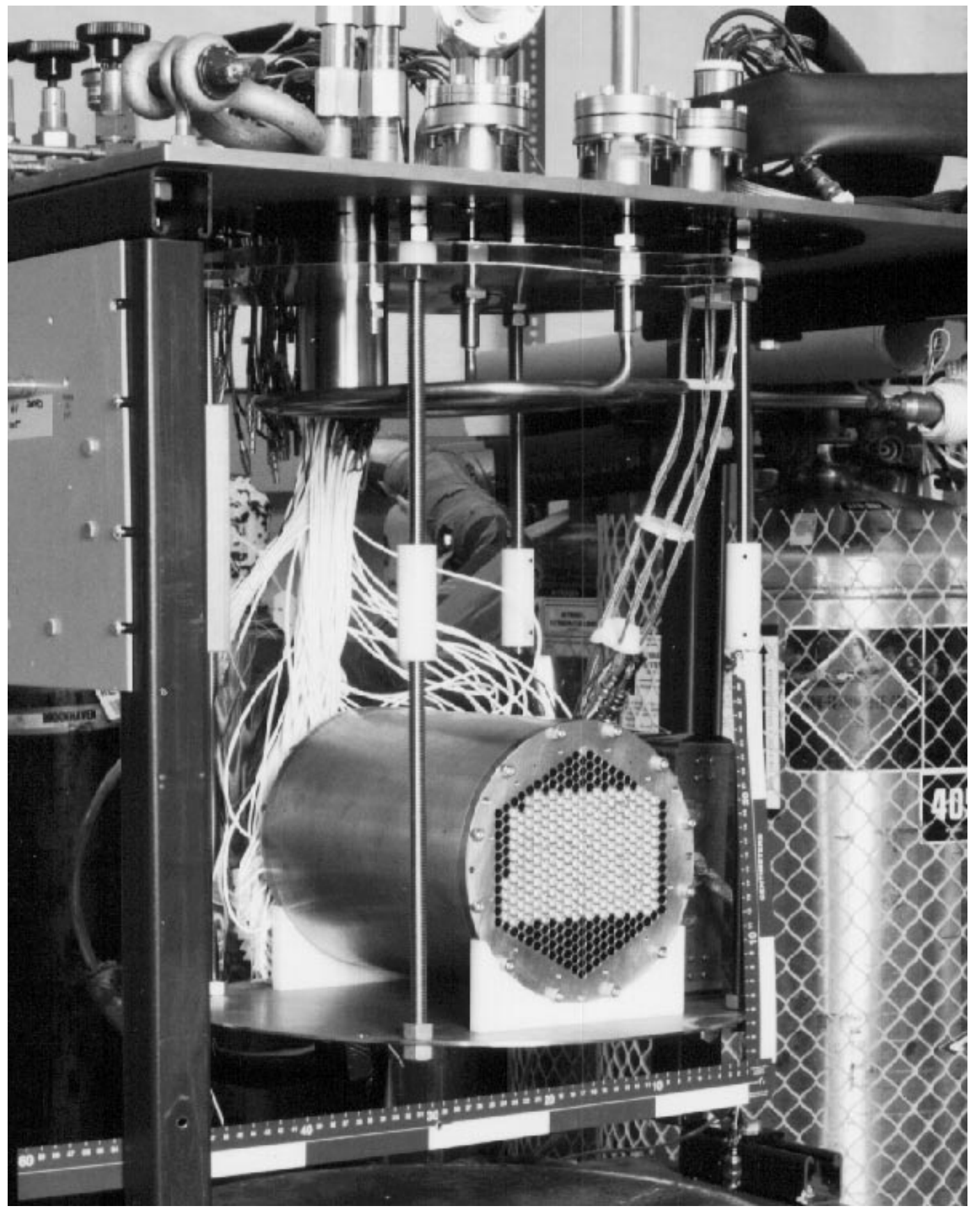

Figure 3: The FCal prototype mounted below the cryostat top plate. The upstream face shows the white plastic caps, while the signal cables are connected to the downstream end of the prototype (scales in $\mathrm{cm}$ ). 


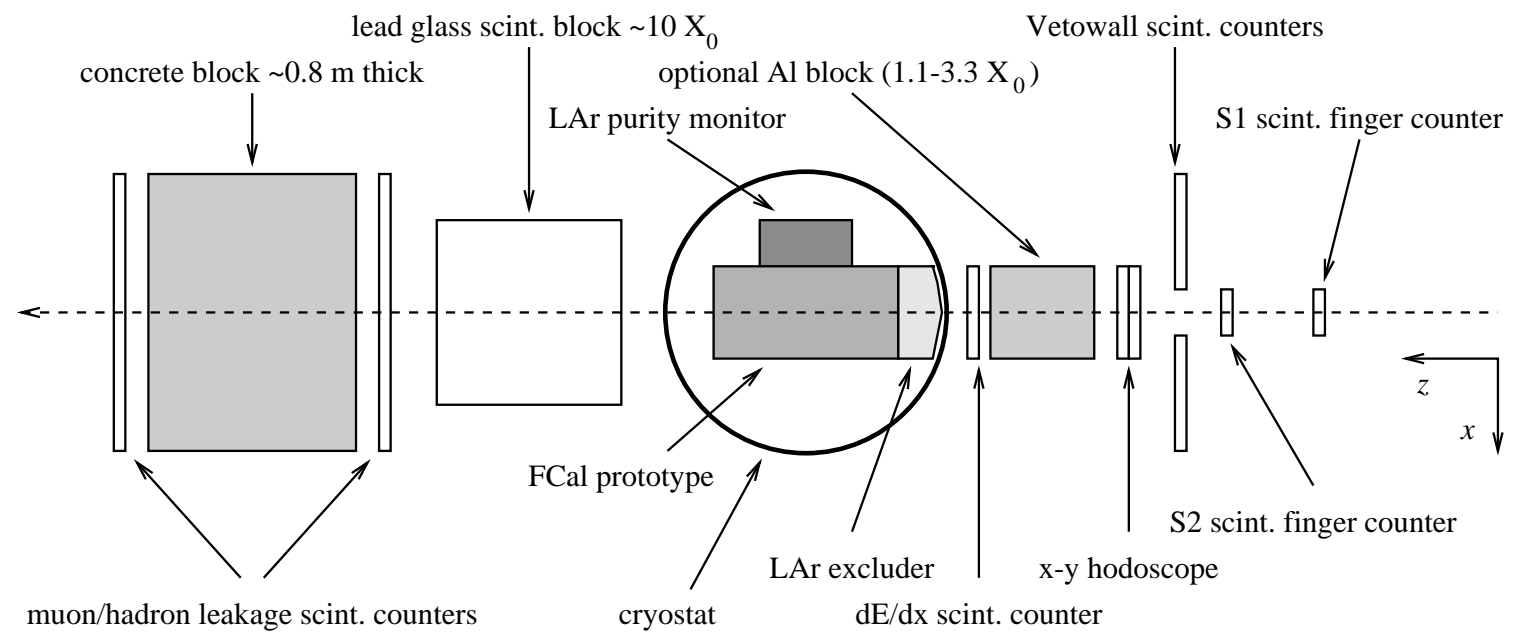

Figure 4: Schematic view of the most important elements in the CERN setup of the FCal test (not to scale).

The hard-disk mentioned above is NFS co-mounted on a DEC 4000/60 workstation, allowing a "quasi-online" reconstruction and analysis program running on this node to access data as it is taken. Therefore a fast analysis of signal quality and system stability is possible during data taking.

\section{Experimental Environments and Simulation}

In this section the experimental environments, i.e. the setup of the testbeams at BNL and CERN, are summarized. The parameters and programs used to simulate the experiments are discussed at the end of the section.

\subsection{Testbeam Setups}

Electron data were taken in two different environments, first in summer 1993 in the B2 testbeam area at the AGS at BNL, with beam energies $E_{\text {beam }}$ available between 2 and $8 \mathrm{GeV}$. In fall 1993 the experiment was moved to the H8 testbeam at the SPS, located in CERN's north area, to take data for $20 \mathrm{GeV} \leq E_{\text {beam }} \leq 200 \mathrm{GeV}$ electrons. Even though both areas have very different experimental conditions, some parts of the setup, like the cryogenic system, the data acquisition (section 2.3), the beam definition counters, and a good part of the trigger system (see following section), were identical.

The cryogenic system consists of a cylindrical cryostat to house the prototype and the LAr monitor box, and a cooling system based on an external liquid nitrogen $\left(\mathrm{LN}_{2}\right)$ supply line and a coiled copper heat exchanger underneath the top plate of the cryostat. The whole system operates at a slight overpressure inside the cryostat, with the $\mathrm{LN}_{2}$ liquefying 
gaseous argon in the cool-down phase, and then maintaining the temperature in the cold phase inside the cryostat. Two temperature probes are installed inside the cryostat as LAr level indicators. Their temperature readings and the pressure are monitored by the data acquisition system. About 150 liters of $\mathrm{LN}_{2}$ per day are needed to keep the temperature and level of the approximately 40 liters of LAr constant.

The unavoidable amount of inactive material in front of the sensitive volume of the FCal prototype, including the cryostat walls, a LAr excluder block (see Fig. 4) and the brass frontplate of the prototype module is estimated to about $0.8 X_{0}$.

The whole cryostat, with the pre-amp box fixed on its top plate, was mounted on a turntable, allowing rotations relative to the beam direction. This then sat on a larger table allowing horizontal and vertical movements with respect to the beam.

As already mentioned in section 2.2, the pre-amp gain had to be adjusted for the different beam energy ranges. Otherwise the analog electronics and the following read-out chain was the same in both setups. It must be mentioned, though, that in both run periods a certain number of electronic channels had to be switched off, mainly due to unacceptable noise levels in the BNL setup, and broken track \& hold units at CERN. The first problem occured mainly because the pre-amps were operated with a very high gain for the relatively small and, for this particular calorimeter, very untypical energy regime. This puts a limit to the BNL data quality in general. In any case channels could be re-arranged to have the remaining sensitive volume (about $50 \%$ at BNL and $75 \%$ at CERN) large enough to laterally contain electromagnetic showers at a sufficient level (around $94-97 \%$, see section 5.1).

\subsection{Beam Definition and Triggers}

The main elements of the CERN beam line are sketched in Fig. 4; the setup at BNL looked very much the same with respect to these parts.

The most upstream counters $\mathrm{S} 1$ and $\mathrm{S} 2$ are small scintillator finger counters, defining a basic beam window of about $2 \times 2 \mathrm{~cm}^{2}$. These two counters, together with the following veto wall scintillator array, form the basic beam trigger: a valid particle trigger is defined by signal coincidence in $\mathrm{S} 1$ and $\mathrm{S} 2$ and no signal in the veto wall.

The $x$-y hodoscope located behind the vetowall consists of $1 \mathrm{~mm}$ wide scintillating fibers, of which 28 are measuring the horizontal $(x)$ and 12 measuring the vertical $(y)$ beam position. This device determines the location of incoming particles in both directions with a resolution of better than $0.5 \mathrm{~mm}$, as the individual fibers for each orientation are partially overlapping with their neighbors.

The aluminum material shown in Fig. 4 is optional. Several configurations have been studied mainly at CERN, with varying material thicknesses between $1 X_{0}$ and $3 X_{0}$. The so-called $d E / d x$ scintillator counter allows selection of electrons with or without interaction before entering the cryostat.

The lead glass block located behind the cryostat is used to measure the longitudinal energy leakage for electrons, especially at higher beam energies. The leakage counter array directly behind the lead glass aids in reducing the pion contamination in the electron event sample, 
while the muon counter arrays located behind a concrete block of about $80 \mathrm{~cm}$ thickness suppress beam muon contributions.

In both setups the beam optics could be adjusted to more or less pure electron beams, with a significant muon and pion background in the CERN beams for $E_{\text {beam }} \geq 100 \mathrm{GeV}$. Electron identification had to be performed offline using signals from the counters described above, to allow for clean event samples.

The trigger not only provided a fast beam definition as mentioned above, but also empty and pulsed events to measure electronics noise and monitor the stability of the electronic chain during normal data taking. Early and late pile-up vetos are used to avoid pileup at high rates. No severe deadtime problems have been observed, even at the high event rate at CERN (few hundred valid particle triggers per second).

\subsection{Simulations}

The GEANT 3.15/90 [6] framework with the standard electromagnetic shower description is used to simulate the electron testbeam runs. Electrons, positrons, and photons are tracked down to the lowest possible energies within GEANT (10 keV), to avoid artificial enhancements of the transition effect [7] in the thin gaps.

The setup of the FCal prototype in the simulation program includes a detailed description of the hardware, including all details of the rod and tube assembly (see section 2.1). In addition, the cryostat wall is included, but no other elements of the beam line except possible additional absorber material. The experimental beam spreads of $\sigma_{x} \approx 2.7(0.5) \mathrm{cm}$ horizontally, $\sigma_{y} \approx 0.75(0.5) \mathrm{cm}$ vertically at BNL (CERN), including phase space limitations by the experimental trigger acceptances defined by $\mathrm{S} 1$ and $\mathrm{S} 2$, have been taken into account with the assumption of gaussian shaped beams. Particle momentum resolution (typically better than $1 \%$ ) has also been included in the simulation.

The simulation signal is defined as the energy deposited in the active liquid argon gap (visible energy). It is stored in the finest possible granularity, i.e. the smallest sensitive unit consisting of one tube and rod assembly. Suppression of units which are not read out in the experiment and collection of the simulation signal into cells corresponding to the electronics channels is done in the reconstruction step, see section 4.2. This allows, for example, shower shape and space resolution studies for various ganging schemes without time-consuming re-runs of simulations. A straight forward reduction of the global sensitive volume in the simulation to the one actually available in the experiment can also be achieved this way.

\section{Signal Reconstruction}

The reconstruction of the signal in the FCal prototype is performed by converting the digital signal from the experiment to an absolute charge, using parameters obtained from an electronic calibration procedure and some corrections for crosstalk and charge collection inefficiencies. The corrections are necessary to unfold some of the influences on the signal which 


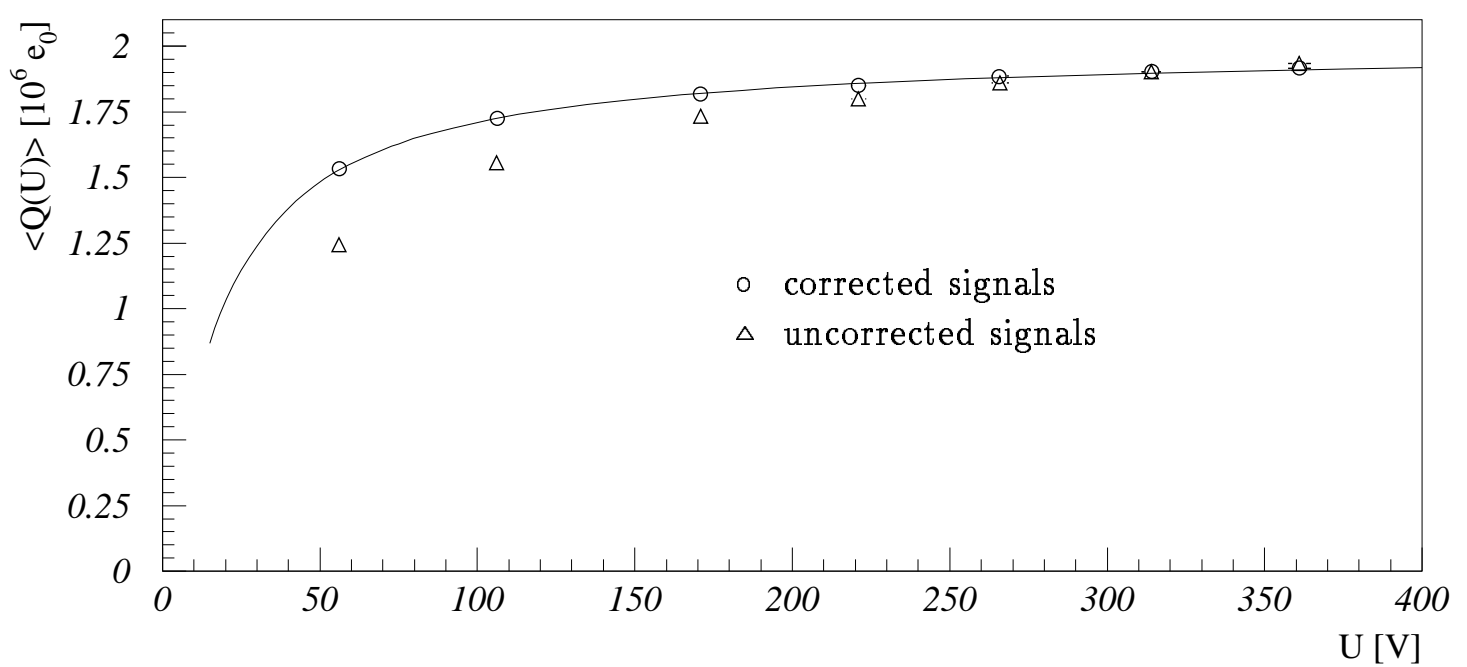

Figure 5: High voltage curve for $8 \mathrm{GeV}$ electrons in the FCal prototype. Shown is the average signal $\langle Q(U)\rangle$ in units of the electron charge $e_{0}$, as function of the voltage $U$ applied across the LAr gap, with and without corrections for the ballistic effect (see text). The line shows the result of a fit to the corrected signals.

are not connected to the calorimeter itself but to the changing environments at testbeams, like crosstalk in cables.

In case of simulated data the energy deposited in the active LAr gap must be converted to the same charge scale, taking into account residual inefficiencies which cannot be corrected in the experimental data, like electronic noise and signal losses due to broken electronic channels. This is certainly only possible up to a remaining finite systematic uncertainty defined by the comparison of data and simulations. The method for including contributions from electronic noise into the simulated response, independent of the shape of the noise distribution itself, is discussed in section 4.2 .

\subsection{Experimental Charge Scale}

The conversion from a measured digital signal $a_{i}$ to an absolute charge $q_{i}$ must be performed in every electronic channel $i$ of the $\mathrm{FCal}$ prototype, using the following relation

$$
q_{i}=s_{i} \cdot\left(a_{i}-p_{i}\right)
$$

where $p_{i}$ is the ADC pedestal for this channel and $s_{i}$ a conversion factor. This linear relation was found to be sufficient for the full dynamic range in both testbeam experiments.

Obviously the pedestals $p_{i}$ and the conversion factors $s_{i}$ have to be determined for each electronic channel. While the pedestals can be measured using randomly triggered empty events during normal data taking, special data with known charge pulses of various heights, fed directly into the pre-amps, are used to measure $s_{i}$. A pattern of 16 non-adjacent channels 
is pulsed simultaneously in one such event. This allows intrinsic correction for crosstalk appearing at the detector level, but leaves the signal uncorrected for possible crosstalk on the pre-amp motherboard and the following chain.

The differences in the shapes of charge pulses originating from ionizations in the LAr and the ones directly fed into the pre-amp lead to an additional ballistic effect, because the later pulses are distorted by reflections. This effect has been calculated in detail and $s_{i}$ has been corrected accordingly, channel by channel, for the BNL data. For the CERN data the $s_{i}$ 's have been measured in the laboratory by explicitly injecting a well-known charge at the detector end of the signal line.

Crosstalk of the order of a few percent between adjacent electronic channels has been observed in CERN data. The amount of this signal enhancement or reduction in a certain channel induced by signals in all other channels has been determined and a full 96-by-96 channel correction matrix was calculated and applied to the experimental signal.

Charge collection inefficiencies due to impurities in the LAr can be measured by varying the electric field in the gap. Fig. 5 shows the response of the $\mathrm{FCal}$ prototype to $8 \mathrm{GeV}$ electrons at $\mathrm{BNL}$, as a function of the voltage applied across the argon gap. Especially at the lowest voltages a non-linear drift time correction following the signal shaping function must be applied, since the ballistic effect increases due to the fact that the drift time becomes larger than the shaping time (compare corrected and uncorrected responses in Fig. 5).

The fitted impurity of about $0.6 \mathrm{ppm}$ oxygen, using the model described in [8], is in very good agreement with independent measurements from an oxymeter and the radioactive sources. From this the absolute charge efficiency was found to be about $96 \%$ and stable during the whole run period. About the same small amount of oxygen is found for the CERN runs, leading to about the same charge collection efficiency.

Finally, a cylindrical cluster algorithm is used to collect the total signal $Q$ associated with the actual shower, and to reduce additional fluctuations introduced by pure noise contributions to this signal:

$$
Q=\sum_{i=1}^{N_{c l}} w_{i} \cdot q_{i} \quad \text { with } \quad w_{i}=\sqrt{\frac{A_{c l, i}}{A_{i}}}
$$

The weights $w_{i}$ applied to each corrected charge signal $q_{i}$ in the $N_{c l}$ channels collected into the cluster are determined by the ratio of the area covered by the cluster $A_{c l, i}$ and the total area $A_{i}$ of this channel ( 1 for central channels in the cluster, $<1$ for channels at the edges, 0 for channels outside).

\subsection{Simulated Response}

The general problem in the comparison of experimental data to simulations is the incorporation of noise into the Monte Carlo. This is mainly important to describe experimental energy- and space resolution and shower shapes. As noise measured by fluctuations of the signal in individual channels in randomly triggered empty events (pedestal fluctuations) does not always follow an easy to model distribution, it is added event-by-event to the simulated 
response, on a channel-by-channel basis, following the method described in [9]. To do this a common scale for the simulation and the experimental signal must be used; here the charge scale, as defined by eq. (1) for experimental data, is taken.

The signal $q_{i}^{m c}$ on the charge scale for simulated data in a volume corresponding to the electronic channel $i$ in the detector can then be expressed as

$$
q_{i}^{m c}=\frac{n_{e}}{2} \cdot e_{0}+q^{n o i s e, i} \quad \text { with } \quad n_{e}=\frac{1}{E_{i o n}} \cdot \sum_{j=1}^{N_{i}} E_{i}^{v i s, j}
$$

where $N_{i}$ is the number of electrodes per electronics channel $\left(N_{i}=2\right.$ for the present setup), $n_{e}$ the number of ionization electrons in the active LAr gap for each electronic channel, $E_{i}^{v i s, j}$ the visible energy deposited in the gap, $E_{\text {ion }}=24.4 \mathrm{eV}$ the ionization energy of LAr [10], and $e_{0}$ the electron charge. $q^{\text {noise, } i}$ is the fully corrected signal of an empty event in this channel, with the general property that its global contribution to the average signal collected from $N_{e v t}$ simulated particle events is vanishing at any given beam energy:

$$
\sum_{k=1}^{N_{e v t}} q_{k}^{n o i s e, i}=0
$$

This is mainly true because the pedestals $p_{i}$ have been determined rather model independently by simple statistical averages on slightly truncated random event samples, to avoid shifts due to very few events with exceptionally large or small pedestals.

Possible signal losses due to the limited lateral acceptance and problematic electronic channels have been taken into account in the reconstruction of the simulated signals by excluding the corresponding areas from the signal sums. The same cluster algorithm used for experimental data is applied for direct comparisons.

\section{Results}

The results obtained in both testbeam runs are summarized in this section.

Data have been taken for electrons in the energy range from 2 to $200 \mathrm{GeV}$, at impact angles of $0.5^{\circ}, 1.2^{\circ}$ and $3.4^{\circ}$. Additional material has been put in the beam line for some runs to simulate the extra material typically mounted in front of a forward calorimeter (beamline elements, cryostat walls, inner detector components). Aluminum blocks of 1.1 (total 1.9) $X_{0}, 2.2(3.0) X_{0}$ and 3.3(4.1) $X_{0}$ depth have been used, with the total amounts including the unavoidable $0.8 X_{0}$ in front of the module (see section 3.1).

\subsection{Linearity of Electron Response}

One of the primary and important aspects to be examined for any electromagnetic calorimeter is the linearity of the electron signal with respect to the beam energy $E_{\text {beam }}$. To study this basic requirement for a module with only limited longitudinal acceptance - such as the 

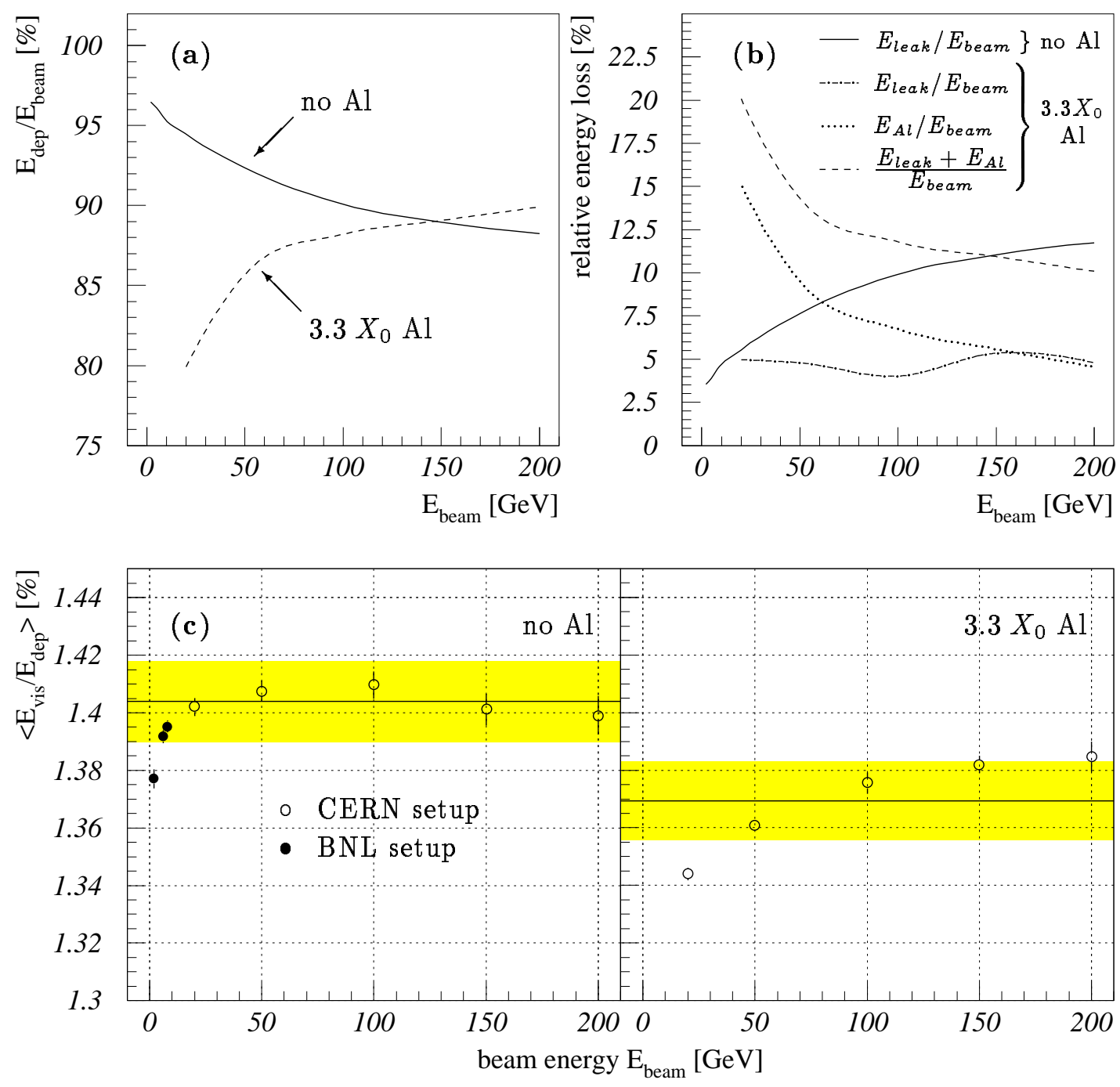

Figure 6: Simulated acceptance of the FCal prototype, as measured by the ratio of deposited energy $E_{\text {dep }}$ to beam energy $E_{\text {beam }}$, without and with $3.3 X_{0}$ aluminum in front of the module ( $a$ ). The fractional energy losses due to leakage and unavoidable upstream material ( $E_{\text {leak }} / E_{\text {beam }}$ ) and due to the extra material $E_{A l} / E_{\text {beam }}$, also obtained from Monte Carlo, are shown in (b). Expectations for signal linearity of an idealized prototype, again from simulations and expressed by the average sampling fraction $\left\langle E_{v i s} / E_{\text {dep }}\right\rangle$ are displayed in (c) for the different setups. The shaded areas indicate the $\pm 1 \%$ range around the average, respectively (errors are statistical only). 
FCal prototype discussed in this note - is certainly not straight forward: the amount of energy deposited in the sensitive volume, which can be sampled and converted to a signal, shows a more non-linear behaviour with respect to $E_{\text {beam }}$ than expected for a module with full longitudinal acceptance. In previous studies signals from the lead glass counter located behind the calorimeter module have been used to correct for this longitudinal energy leakage $[11,12]$. Here we consider a different method using acceptance corrections from simulations, which also allows for unfolding signal distortions introduced by the testbeam environment such as energy losses in material in front of the calorimeter module.

The acceptance of the sensitive volume of the FCal prototype, i.e. the volume filled with tube/rod assemblies, can be expressed as the ratio of energy deposited inside this volume $E_{d e p}$ to the beam energy $E_{\text {beam }}$. It is known to be a non-linear function of $E_{\text {beam }}$ and is shown in Fig. 6(a). From the same figure it is obvious that a significant amount of inactive material in front of the sensitive volume changes the beam energy dependence of $E_{\text {dep }} / E_{\text {beam }}$ severely, due to the extra energy losses and the earlier shower starting point. Fig. 6(b) shows the beam energy dependence of the two major contributions to energy losses in the different setups, longitudinal leakage and the loss in the inactive material in front. The lateral energy leakage is found to be negligible ( $\ll 1 \%$ of $E_{\text {beam }}$ ) for all studied setups.

The Monte Carlo prediction for the linearity of the electron signal in the prototype is shown in Fig. 6(c), for the setup without and with extra material in the beam. In this case the variation of the sampling fraction $E_{v i s} / E_{d e p}$, with $E_{v i s}$ being the energy deposited in the liquid argon, is a measure of linearity with the previously discussed acceptance problems unfolded. The average $\left\langle E_{v i s} / E_{d e p}\right\rangle$ shown in the figure is calculated using signals for $20 \mathrm{GeV} \leq$ $E_{\text {beam }} \leq 200 \mathrm{GeV}$ only, as the sampling fraction at lower energies is certainly more effected by the unavoidable dead material (cryostat wall) in front of the calorimeter. Another influence can come from the chosen particle tracking cuts in the simulation, which at the lower energies may still introduce an enhancement of the already mentioned transition effect (section 3.3).

The measured response of a calorimeter module with limited containment for electromagnetic showers can be understood with respect to electron signal linearity in at least two ways. First, the simulated signal inside this module can be compared to the corresponding measured signal if its reconstruction includes all known experimental inefficiencies such as fiducial volumes, broken electronic channels, and noise (see section 4.2). The average relative difference between these two signals, determined at various beam energies, then defines the residual experimental deviation from linearity for an intrinsically linear calorimeter design [9]. Fig. 7 shows good agreement between the experimental and simulated energy response distributions for the FCal prototype. The absolute experimental charge scale can presently be understood from the comparison of the average response from data and simulation for various beam energies to about $5 \%$.

Another approach, which has the advantage of being independent of the experimental charge scale and the details of the signal simulation, is to use a correction obtained from the curve given in Fig. 6(a) and to apply it to the measured average charge response $\langle Q\rangle$, 

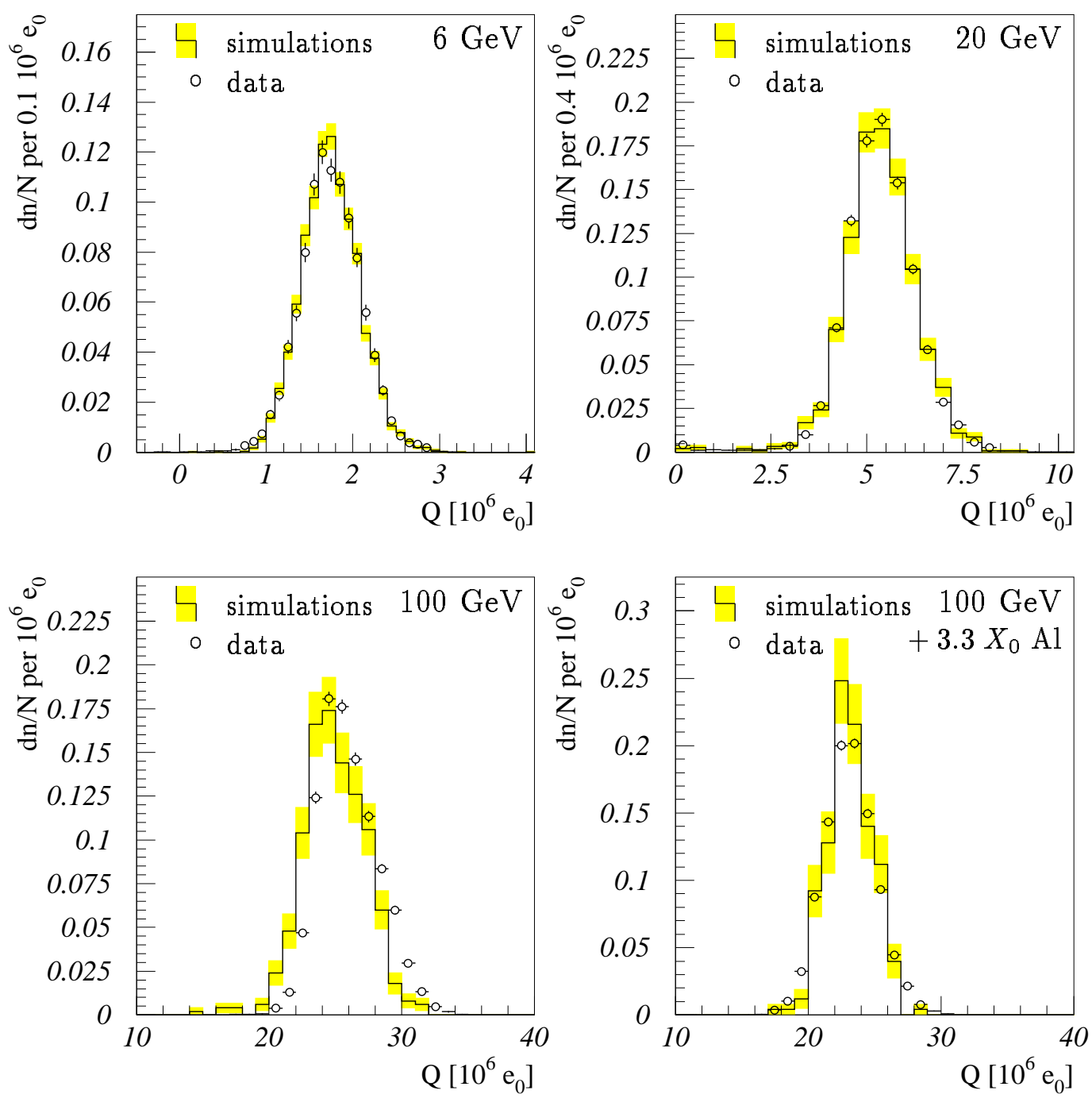

Figure 7: Distributions of the signal $Q$ for electrons at different beam energies, for data and simulations. $Q$ is measured in units of the electron charge $e_{0}$. 


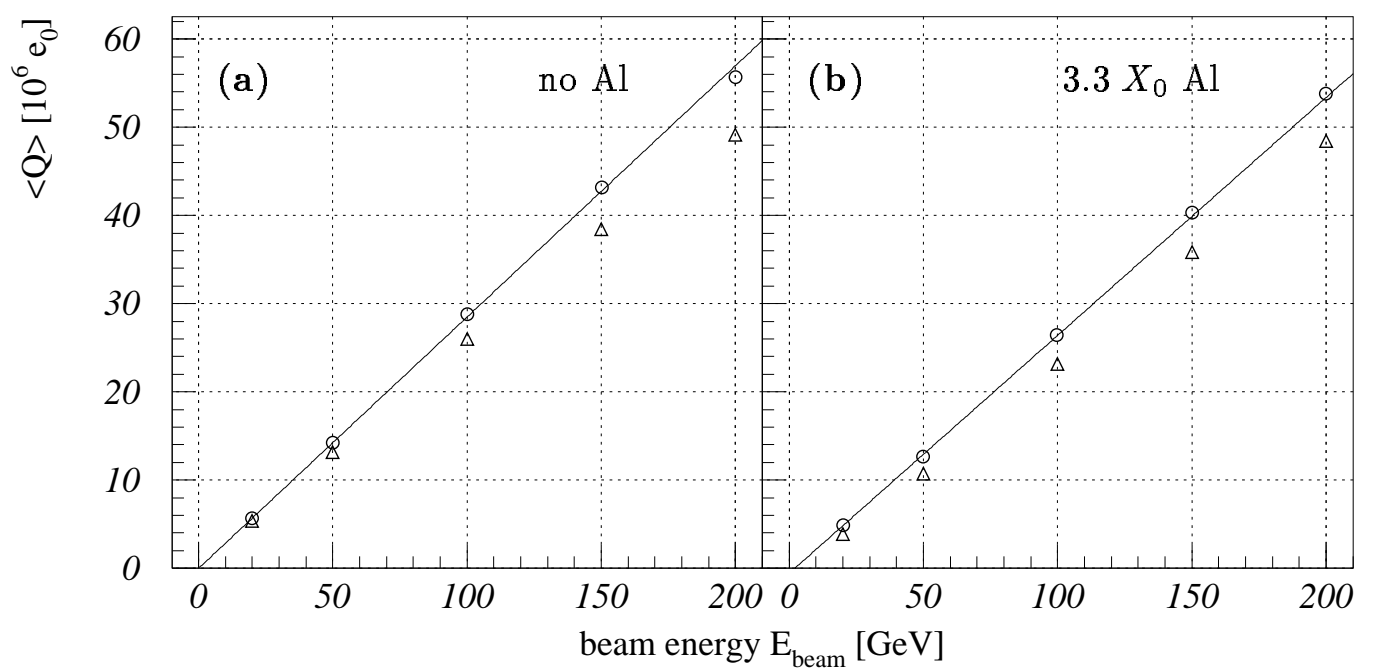

Figure 8: Experimental charge response $\langle Q\rangle$ of the FCal prototype module to electrons at $3.4^{\circ}$, as function of the beam energy $E_{\text {beam }}$. Uncorrected $(\Delta)$ and corrected data ( $(0)$ are shown for the setup without (a) and with (b) extra aluminum in the beam (see eq. (4) and text for details). Statistical errors are smaller than symbol sizes.

calculated as described in section 4.1 :

$$
\left\langle Q_{\text {corr }}\left(E_{\text {beam }}\right)\right\rangle=\left(\frac{E_{\text {beam }}}{E_{\text {dep }}}\right)_{m c} \cdot\left\langle Q\left(E_{\text {beam }}\right)\right\rangle
$$

This correction is only dependent on the quality of the simulated average absorption characteristics of the module, given by $E_{d e p}$. The effect on the signal is shown in Fig. 8 for the CERN data without (a) and with (b) extra material in the beam.

Fig. 9 finally shows the deviation from signal linearity, which in this approach is determined by the remaining beam energy dependence of the relative difference between $Q_{\text {corr }}$ from eq. (4) and the straight line fit shown in Figs. 8(a),(b), respectively. From this the linearity for electrons is found to be within $2 \%$ and certainly within the present Monte Carlo predictions for this calorimeter module. The expected non-linearity for the data with extra material is also reproducible, with some remaining inconsistency at $20 \mathrm{GeV}$. Not taken into account in these figures is a systematic error of about $6 \%$ (conservatively), suggested by the accuracy of the electronic calibration system and absolute comparisons to Monte Carlo.

The variation of the response with the impact angle and additional material in the beam has been studied systematically. It is generally found to be only small $(\lesssim 1 \%)$ for impact angles between $0.5^{\circ}$ and $3.4^{\circ}$, with a small dependence on the amount of inactive material (slightly larger variations with less material, as expected). 


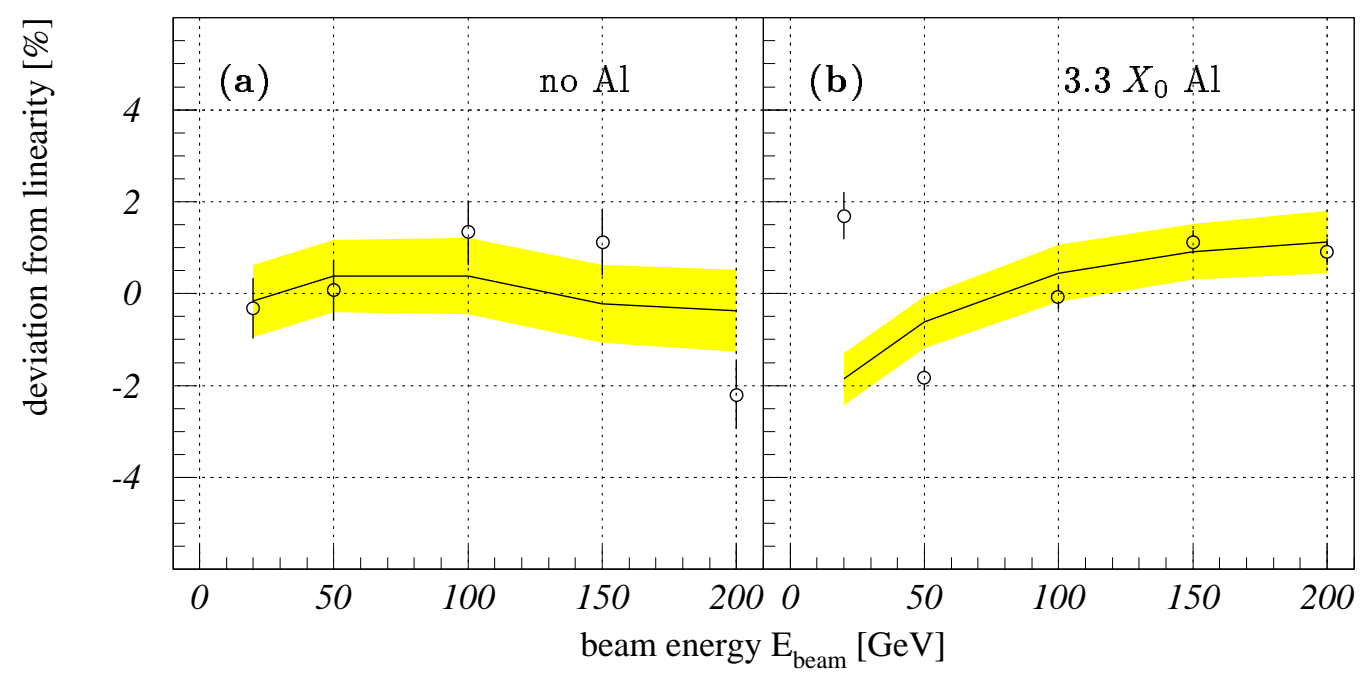

Figure 9: Deviation from linearity for electrons versus beam energy $E_{\text {beam. }}$. Shown is the relative difference between the corrected charge response and the straight line shown in Fig. 8, for data without and with extra material in the beam. The lines and shaded areas indicate the Monte Carlo prediction and its present statistical accuracy (all errors are statistical only).

\subsection{Electron Energy Resolution}

The determination of the next important performance parameter for the calorimeter, the energy resolution, is again limited by the size of the prototype. To extract a realistic estimator, the direct measurement of the energy leaking out of the module into the lead glass counter must be included in the signal reconstruction.

In addition small deviations from gaussian shapes are observed for signal distributions (Fig. 7), especially for the data with no extra material in the beam. This effect is due to the inhomogeneous front face of this calorimeter, resulting in dependence of the signal on the impact point. Obviously this dependence is less severe for events where the beam particle starts to shower in the upstream material. A position-dependent correction is applied which is based on a careful reconstruction of the impact point exclusively from calorimeter signals by means of a multi-parameter likelihood method and signal correction tables parameterized by the impact point coordinates [12].

The average contribution of electronic noise to signal fluctuations in the cluster defining the total signal (see section 4.1 ) has been determined to be $2.24 \mathrm{GeV}(0.23 \mathrm{GeV})$ for CERN (BNL) data. The noise, especially at CERN, is dominated by fluctuations in the data acquisition system, with an almost negligible contribution from the front-end electronics noise (see section 2.2). These anomalously large contributions (at the lower energies) have been unfolded from the measured energy resolutions.

Fig. 10 shows the energy resolution obtained for electrons without and with extra material in the beam. A sampling term of $a=(36.3 \pm 0.3) \% / \sqrt{\mathrm{GeV}}$ and a constant term $c=$ 

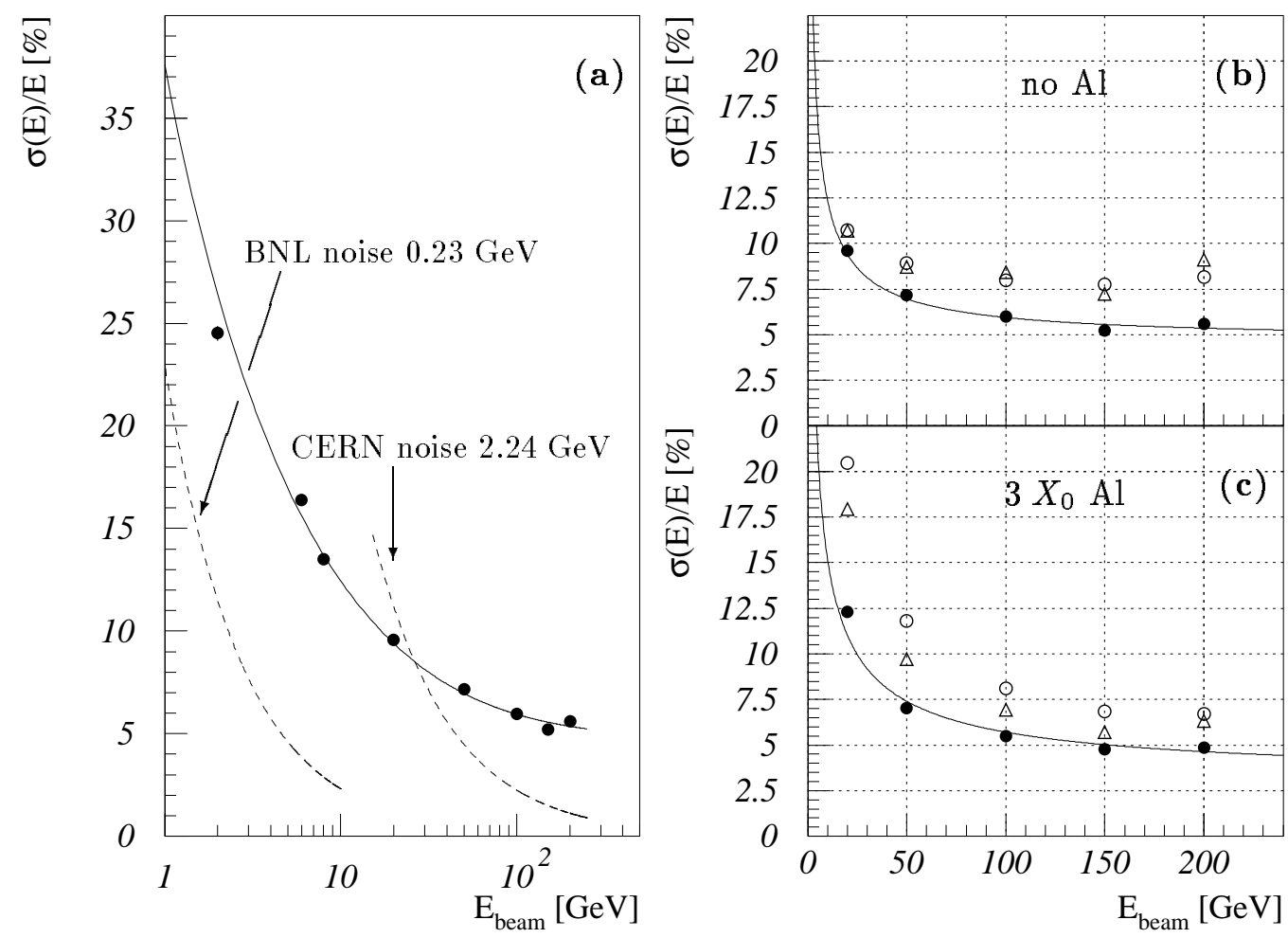

Figure 10: Energy resolution of the prototype module for electrons. (a) shows the fit of eq. (5) to the fully corrected resolution (•) after noise unfolding, for electrons with no extra material in the beam. The same data is also shown in (b), together with the resolution without impact point and leakage correction for Monte Carlo $(\Delta)$ and data $(0$, see text). The energy resolution for data with extra material and the corresponding fit can be found in (c). 
$(4.7 \pm 0.1) \%$ can be found in a common fit of (see [7], for example):

$$
\sigma(E) / E=\sqrt{a^{2} / E_{\text {beam }}+c^{2}}
$$

to BNL and CERN data (Fig. 10(a)). Introducing additional material into the beam increases the sampling term to about $47 \%$, due to the additional shower starting point fluctuations, but decreases the constant term to $c=(3.2 \pm 0.1) \%$, due to better longitudinal containment and reduced sensitivity to the impact point. Compare Figs. 10(b) and (c).

The simulated energy resolution can only be compared to the data without leakage correction, as signals from the lead glass counter are not available in the Monte Carlo. Figs. 10(b),(c) show that the simulations predict the experimental fluctuations quite well, with a systematically better resolution for the setup with extra upstream material.

\subsection{Electron Space Resolution}

The last important performance parameter considered in our test program is the space resolution in both the horizontal $(x)$ and vertical $(y)$ planes. These parameters have been determined via a sophisticated algorithm based on an impact point reconstruction using a multi-parameter likelihood method [12] which explicitly takes into account the lateral electromagnetic shower shapes and the available channel granularity (same as for the signal correction mentioned above). The reconstructed impact point is then compared to the beam position measured in the $x-y$ hodoscope (Fig. 4, section 3.2). The result for an electron impact angle of $3.4^{\circ}$ is shown in Fig. 11, fitted with the following beam energy dependence:

$$
\sigma_{d}=\sqrt{a_{d}^{2} / E_{\text {beam }}+c_{d}^{2}}, \quad d=x, y
$$

The most interesting high energy limits of the space resolution $c_{x}, c_{y}$ are found to be:

$$
c_{x}=\left\{\begin{array}{c}
(0.84 \pm 0.01) \mathrm{mm}(\text { no Al }) \\
(0.79 \pm 0.01) \mathrm{mm}\left(2.2 X_{0} \mathrm{Al}\right) \\
(0.69 \pm 0.01) \mathrm{mm}\left(3.3 X_{0} \mathrm{Al}\right)
\end{array} \quad, \quad c_{y}=\left\{\begin{array}{c}
(0.55 \pm 0.01) \mathrm{mm}(\text { no Al }) \\
(0.50 \pm 0.01) \mathrm{mm}\left(2.2 X_{0} \mathrm{Al}\right) \\
(0.41 \pm 0.02) \mathrm{mm}\left(3.3 X_{0} \mathrm{Al}\right)
\end{array}\right.\right.
$$

The corresponding parameters $a_{x}\left(a_{y}\right)$ fall between $3.8(3.4) \mathrm{mm}$ for the data with no extra material and $7.1(6.4) \mathrm{mm}$ for the data with $3.3 X_{0}$ additional material in the beam. The constant terms $c_{x}, c_{y}$ are not only approaching the experimental resolution limit of the beam position determination set by the $x-y$ hodoscope, but also the Monte Carlo estimators.

The angular dependence of the space resolution can only be observed horizontally, as the module has been rotated along a vertical axis to change the electron impact direction. The horizontal space resolution degrades at larger angles (with some dependence on the extra material thickness) because of coupling from longitudinal shower fluctuations. The horizontal position resolution for $150 \mathrm{GeV}$ electrons is improved by about $30 \%$ going from $3.4^{\circ} \mathrm{impact}$ angle $\left(\sigma_{x}=0.89 \mathrm{~mm}\right)$ to $0.5^{\circ}\left(\sigma_{x}=0.62 \mathrm{~mm}\right)$. Extra material in front softens the sensitivity to the impact angle to $25 \%$. 

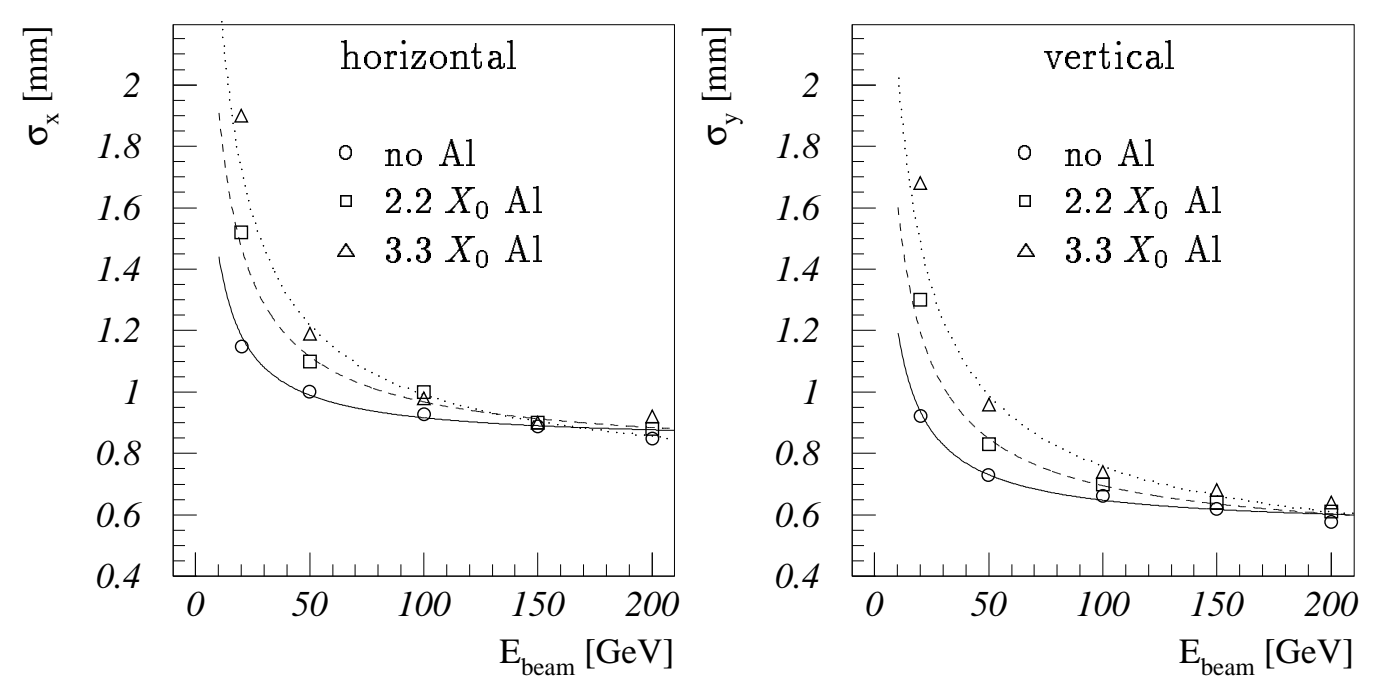

Figure 11: The horizontal $\left(\sigma_{x}\right)$ and vertical $\left(\sigma_{y}\right)$ experimental space resolution for electrons in the FCal prototype, as function of the beam energy $E_{b e a m}$. Results are shown for data without extra material and with two different aluminum thicknesses. The curves indicate fits of eq. (6) to the data points.

\section{Conclusions}

A forward calorimeter at a high luminosity hadron collider is subject to many constraints. Paramount among these, it must operate within the highest particle energies and fluxes of any detector element. Speed of response and radiation resistance are severe challenges and their solutions force some compromises on the design. We have seen that this new approach to liquid argon calorimetry, which was specifically developed for high luminosity applications, also demonstrates excellent calorimetric performance in this difficult regime. This liquid argon calorimeter with novel read out geometry consisting of small tube/rod assemblies forming thin cylindrical shell shaped gaps has been tested with electrons for the first time in 1993 at BNL and CERN. The most important performance requirements are confirmed by the test results, even within the limited acceptance of the prototype module. These include signal linearity of better than $2 \%$ between 20 and $200 \mathrm{GeV}$, and a high energy limit of the energy resolution of about $4 \%$. A space recolution of about $0.6 \mathrm{~mm}$ is achieved in the high energy limit.

Because the forward calorimeter must also operate behind a significant amount of material (tracker and beam line elements), a first experimental test on the performance limitations in such an environment has been conducted by adding additional inactive material in front of the calorimeter module. With $3.3 X_{0}$ of aluminum in the beam, linearity was observed to agree with simulation for a small module, to order of $2 \%$. The energy resolution at high energies improves to about $3 \%$, which is expected due to less sensitivity of the signal to inhomogeneities of the calorimeter front face and better longitudinal containment. Space resolution for highest energies is also slightly improved to about $0.4 \mathrm{~mm}$ in this case. 
Based on the results from this first test and additional extensive engineering and physics performance simulation studies, including radiation shielding properties [13], the calorimeter design presented in this note has been accepted by ATLAS as the baseline for the forward calorimeter.

\section{Acknowledgements}

Many people contributed to the success of these testbeam experiments. We would like to thank H. Gordon, D. Lissauer, V. Radeka, S. Rescia (all at BNL), W. Willis (Columbia University, New York), Y. Kamishkov and V. Khovansky (both ITEP Moscow) for fruitful conversations and help concerning the calorimeter and its electronics; A. Carroll, D. Dayton and all other BNL staff members for helping us during installation and running at BNL. We also appreciate contributions of J. Janesky (University of Arizona), A. Lindenberg (Columbia University, New York) and Z. Zhang (University of New York at Stony Brook).

We also thank D. Fournier (LAL Orsay), D. Froidevaux, and P. Jenni (both CERN), who made the CERN test possible. We also received crucial support from K. Bussmann, K. Elsener, N. Doble and other CERN staff members, and J. Ban and J. Huber (both MPI Munich). Finally we would like to thank M. Eklund (University of Arizona) for help with both the online and offline computing hardware.

\section{References}

[1] ATLAS coll., Technical Proposal for a General-Purpose pp Experiment at the Large Hadron Collider at CERN, CERN/LHCC/94-43 (1994)

[2] for a summary see [1] p. 213ff and references therein

[3] J.P. Rutherfoord, Ion Loading in Liquid Ionization Calorimeters, GEM TN-91-27 (1991)

[4] G.C. Blazey, Monitoring Liquid Argon Purity at DZero, in Proc. of Int. Conf. on Calorimetry in High Energy Physics, Batavia, Ill. Oct. 29 - Nov. 1, 1990 (1991)

[5] R.L. Chase, C. de la Taille, S. Rescia and N. Seguin-Moreau, Transmission lines connection between detector and front end electronics in liquid argon calorimetry, Nucl. Instr. and Meth. A330 (1993) 228-242

R.L. Chase, C. de la Taille and N. Seguin-Moreau, Experimental results on cable-coupled preamplifiers $(\emptyset T), \mathrm{Nucl}$. Instr. and Meth. A343 (1994) 598-605

[6] R. Brun and F. Carminati, GEANT Detector Description and Simulation Tool, CERN Programming Library Long Writeup W5013 (1993)

[7] R. Wigmans, On the energy resolution of uranium and other hadron calorimeters, Nucl. Instr. and Meth. A 259 (1987) 389 
[8] W. Hofmann et al., Production and transport of conduction electrons in a liquid argon ionization chamber, Nucl. Instr. and Meth. 135 (1976) 151

[9] H1 Calorimeter Group (B. Andrieu et al.), Beam tests and calibration of the H1 liquid argon calorimeter with electrons, Nucl. Instr. and Meth. A350 (1994) 57-72

[10] T. Doke, Fundamental Properties of Liquid Argon, Krypton and Xenon as Radiation Detector Media, in Experimental Techniques in High-Energy Nuclear and Particle Physics, ed. T. Ferbel, World Scientific (1991)

[11] M.I. Ferguson et al., A Liquid Argon Forward Calorimeter Prototype: Beam Test Results, ATLAS internal note CAL-NO-042 (1994)

[12] A. Savine et al., Beam Test of Liquid Argon Tube Calorimeter Prototype, Proc. of the $5^{\text {th }}$ Int. Conf. on Calorimetry in High Energy Physics, Brookhaven, New York Sept 25 Oct 1, 1994, ed. Howard A. Gordon and Doris Rueger, World Scientific 1995.

[13] J. Rutherfoord, L. Shaver and M. Shupe, The ATLAS Forward Region, ATLAS internal note CAL-NO-35 (1994) 